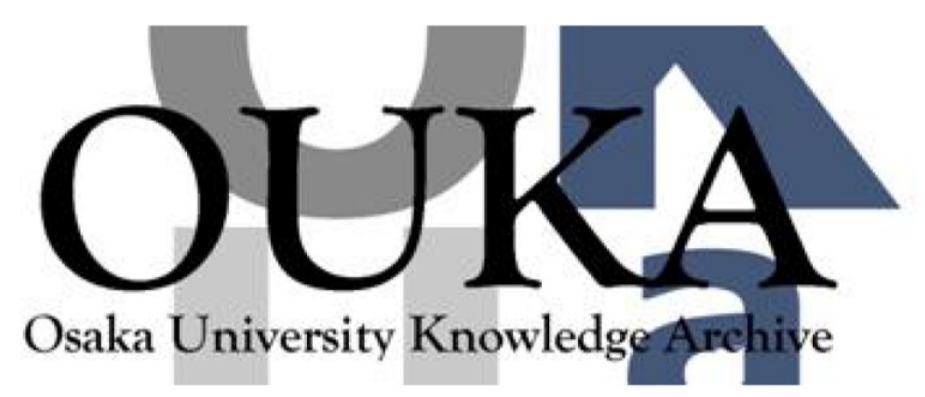

\begin{tabular}{|c|l|}
\hline Title & $\begin{array}{l}\text { Momentum distribution of accelerated ions in } \\
\text { ultra-intense laser-plasma interactions via } \\
\text { neutron spectroscopy }\end{array}$ \\
\hline Author(s) & Habara, H.; Kodama, R.; Sentoku, Y. et al. \\
\hline Citation & Physics of Plasmas. 10(9) p.3712-p.3716 \\
\hline Issue Date & $2003-09$ \\
\hline oaire:version & VoR \\
\hline URL & https://hdl. handle. net/11094/3334 \\
\hline rights & \\
\hline Note & \\
\hline
\end{tabular}

Osaka University Knowledge Archive : OUKA

https://ir. Library. osaka-u. ac. jp/

Osaka University 


\title{
Momentum distribution of accelerated ions in ultra-intense laser-plasma interactions via neutron spectroscopy
}

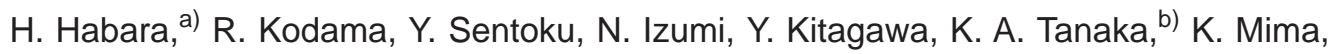 \\ and T. Yamanaka \\ Institute of Laser Engineering, Osaka University, Suita 565-0871, Osaka, Japan
}

(Received 2 January 2003; accepted 27 May 2003)

\begin{abstract}
The dependence of neutron spectra on the incident laser polarization has been studied to elucidate ion acceleration processes in ultra-intense laser-plasma interactions. Obliquely incident, 50-100 TW (450-800 fs) laser pulses irradiated a deuterated plastic solid target at $10^{19} \mathrm{~W} / \mathrm{cm}^{2}$ with both $s$ and $p$-polarizations. Neutron spectra from the deuteron-deuteron $d(d, n)^{3} \mathrm{He}$ nuclear reactions show the ion acceleration into the target in the direction of the target normal for both polarizations. The experiment and particle-in-cell simulations indicate the ions are accelerated inside the target by an intense electrostatic field created by charge displacement at the laser focus. (C) 2003 American Institute of Physics. [DOI: 10.1063/1.1593650]
\end{abstract}

\section{INTRODUCTION}

Recent progress of the chirped pulse amplification technique has allowed access to relativistic laser intensities (i.e., where the quiver velocity of electrons in the laser field approaches the speed of light). At $10^{19} \mathrm{~W} / \mathrm{cm}^{2}$, for example, the gamma parameter of the quivering electrons exceeds 2 . Such intense laser pulses can generate energetic electrons ${ }^{1,2}$ and ions ${ }^{3}$ in the $\mathrm{MeV}$ range. These energetic particles are extremely important in the context of the fast ignitor $(\mathrm{FI})^{4}$ scheme in inertial confinement fusion research, as well as in many applications, such as nuclear physics and laboratoryastrophysics.

One of the most important parameters in FI physics is the energy coupling efficiency from the laser pulse to the energetic particles. The conversion efficiency to hot electrons is estimated about $30 \%-50 \%$ from direct and indirect measurements in laser plasma experiments. ${ }^{1,5}$ On the other hand, the conversion efficiency to ions has not been well evaluated yet because of the difficulty of measuring the ion momentum distribution due to the complex acceleration of ions, e.g., the acceleration at front and at rear separately for planar geometry. Direct observation of ions, such as track detectors placed some distance from the target, cannot measure only those ions accelerated at the interaction point, which may be of some importance in the FI scenario.

It is also important to know the momentum distribution of laser-accelerated ions to evaluate the acceleration mechanisms in laser-plasma interactions. Recently, with successful results in simulations and experiments, it has become clear that, at the rear side of target, ions are accelerated by an electrostatic field due to the sheath potential between the escaping electrons and the ions. ${ }^{6,7}$ On the other hand, it is not clear which mechanism is dominant at the front surface of the target. Several possibilities are reported, such as Cou-

\footnotetext{
a) Present address: Central Laser Facility, Rutherford Appleton Laboratory, Chilton, Didcot, Oxfordshire OX11 0QX, UK.

${ }^{b}$ Faculty of Engineering, Osaka University, Suita 565-0871, Osaka, Japan.
}

lomb expansion, ${ }^{8}$ the static field acceleration (which is similar to the rear surface acceleration) and shock wave acceleration. ${ }^{9}$ Measurements of the momentum distribution of ions are crucial to help identify these ion acceleration mechanisms.

In this paper, the momentum distribution of accelerated ions in ultra-intense laser interactions is presented for the first time. The ion distributions were unfolded from neutron spectra generated by nuclear reactions of the accelerated deuterons with target deuterons (beam-type fusion). Compared with direct measurements of ions using track detectors, neutrons have an advantage because they are independent from the strong electric and magnetic field created in the over-dense plasma. ${ }^{10,11}$ The neutron spectra were detected from different viewing angles using large area neutron spectrometers and were analyzed using a Monte Carlo simulation tool. The experimental results have been also compared with 2D particle-in-cell (PIC) simulations to understand ion acceleration.

\section{EXPERIMENT}

The experiments were performed using the 30TW Gekko MII $^{12}$ and 100TW Gekko XII ${ }^{13}$ short pulse laser system with glass amplifiers at the Institute of Laser Engineering (ILE), Osaka University. The laser energies were $20-50 \mathrm{~J}$ on target for $1.05 \mu \mathrm{m}$ laser light and the pulse widths were $450-800$ fs. The leakage prepulse level was monitored with a pin diode to be less than $10^{-4}$ to the main pulse intensity and mostly appeared at 700 ps before the main pulse. Two different linear polarizations, i.e., s-polarization and $p$-polarization, were used to obtain the polarization dependence of the neutron spectra from $d(d, n)^{3} \mathrm{He}$ fusion reaction. A $5 \mu \mathrm{m}$ thickness deuterated plastic (CD) target was obliquely irradiated by the short pulse laser light through a f/3.8 off-axis parabolic focusing mirror. The peak intensity of the main pulse on the target was $10^{19} \mathrm{~W} / \mathrm{cm}^{2}$ from the spot of $30 \mu \mathrm{m}$ in a diameter obtained with a x-ray pinhole camera. 
Neutron spectra were detected with neutron spectrometers that consisted of plastic scintillators combined with a photomultiplier-tube through the time of flight (TOF) technique. Two different types of the spectrometer were used in the experiment: One type was a single channel detector used to obtain the spectra from a temporal current profile of the scintillator output, and another a large area multichannel detector system to obtain the spectra from the hit channel number. ${ }^{14}$ Reliable detection ranges on neutron energies of the system were under $20 \mathrm{MeV}$. The neutron energy resolutions were about $29 \mathrm{keV}$ (multichannel detector) and 110 $\mathrm{keV}$ (single channel detector). Three detectors (one is the current type and two the counting type) were set up at different view angles to obtain the angular distribution of neutron spectra. All the neutron detectors had $5 \mathrm{~cm}$-thick cylindrical polyethylene collimators that surrounded $5 \mathrm{~mm}$-thick lead tubes. These collimators were placed inside the chamber to reduce gamma-ray noise from the scattered electrons, e.g., at the chamber wall and from the other diagnostics such as pinhole cameras. In order to reduce the gamma noise from the target itself, 15 and $5 \mathrm{~cm}$ lead blocks were placed at the front and at the side of the detectors. The effects of neutron scattering due to the lead blocks were characterized with thermal neutrons generated in imploded glass micro-balloon (GMB) with deuteron gas fuel-resulting in a negligible scattering effect on the spectra.

The momentum distribution of accelerated ions was evaluated through a Monte Carlo simulation in threedimensional (3D) momentum space. The calculated spectra were compared with all the experimental spectra detected at different view angles. In the calculation, the target is assumed to be a solid density $\left(\sim 1.1 \mathrm{~g} / \mathrm{cm}^{-3}\right)$ deuterated plastic (polyethylene) plane $(100 \times 100 \times 5 \mu \mathrm{m})$ with an initial temperature of $0.1 \mathrm{eV}$ (not ionized). Test particle numbers are 8192. The stopping power to the ions is given by Jackson's equation ${ }^{15}$ taking account of density effects in the relativistic region on scattering and energy losses. Cross sections for the neutron reaction between deuterons were taken from established data. ${ }^{16}$ In the calculation, an initial momentum distribution is required. A number of momentum distributions, for example, monoenegetic, Maxwellian, twotemperature Maxwellian, isotropic, anisotropic, and so on, were tested to fit the output spectra to all the experimental neutron spectra obtained simultaneously at different view angles.

\section{RESULTS}

Solid lines in Figs. 1(a) and 1(b) show the observed neutron spectra detected at (a) $56^{\circ}$ and (b) $39^{\circ}$ to the target normal, corresponding to the opposite side to the laser incidence direction. The laser irradiated the target with $40^{\circ}$ angles to the target normal as the $s$-polarization condition. Similarly, the spectra which were generated by nearly $p$-polarized light with $36^{\circ}$ incident angle (also including 18 deg. $s$-component) are shown in the solid lines of Figs. 2(a) and 2 (b), taken at $56^{\circ}$ and at target normal, respectively. The

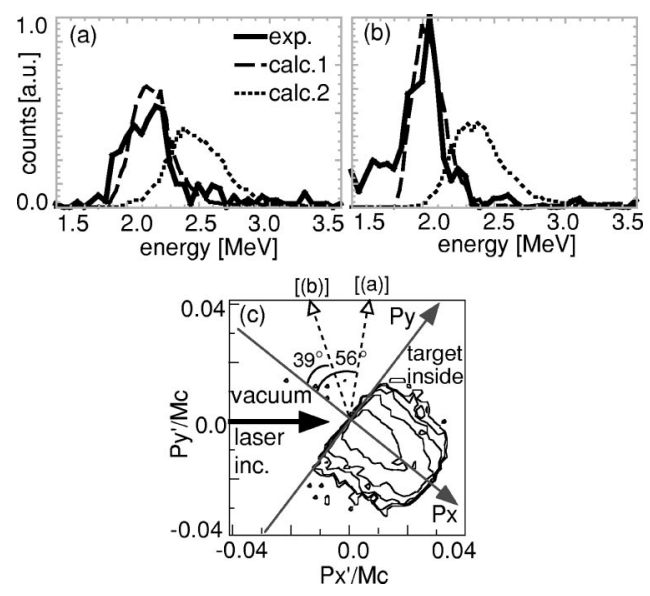

FIG. 1. The neutron spectra at $s$-polarization detected at (a) $56^{\circ}$ and (b) $39^{\circ}$ to the target normal. The experimental spectra are shown by solid lines. Dashed lines and dotted lines represent the calculated spectra from the 3D Monte Carlo code assuming the ion acceleration in the direction of the target normal and the laser direction, respectively. (c) Contour plot of twodimensional distribution of the ion momentum obtained by spectral fitting as shown by the dot-dashed line in (a) and (b). The momentum is normalized by Mc (M: Ion rest mass, c: Light speed). Each contour line represents every $10^{0.2}$ of the ion number in a logarithmic scale.

signal levels on the figures are normalized to be a neutron yield per solid angles taking account of the detection efficiency of each detector.

All the spectra indicate Doppler shift on the peak to the lower energy from $2.45 \mathrm{MeV}$ given by deuteron-deuteron (D-D) thermal fusion reaction. These spectral shifts are strong evidence that the ions were accelerated into the target inside for either polarization conditions from the momentum conservation between the accelerated and the target deuterons.

Detailed analysis of the ion motion has been performed with the 3D Monte Carlo simulation to fit the calculated spectra to the experimental results obtained at all directions. The dashed lines in Figs. 1(a), 1(b), 2(a), and 2(b) show the
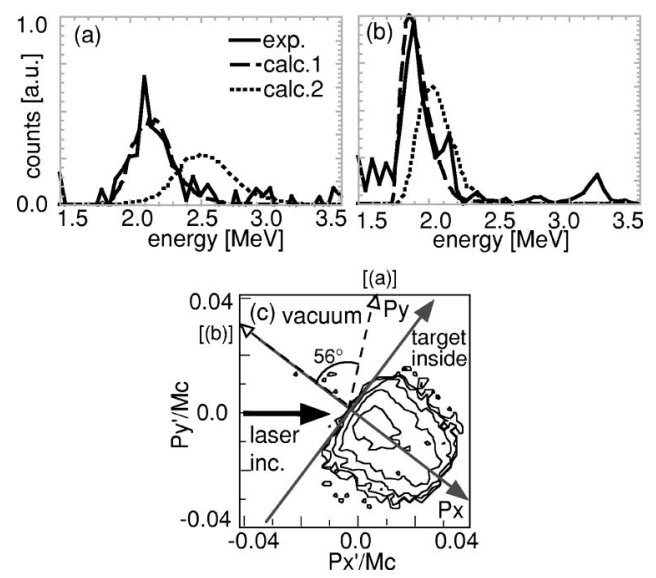

FIG. 2. The neutron spectra at $p$-polarization detected at (a) $56^{\circ}$ to the target normal and at (b) the target normal. Solid lines are experimental spectra. Dashed lines and dotted lines show the calculated spectra corresponding to the ion acceleration to the target normal and to the laser direction, respectively. (c) Contour plot of 2D spatial distribution of the ion momentum for the well-fitted calculation as shown by the dot-dashed line. The other figure conditions are as same as Fig. 1(c). 
well-fitted spectra, giving the ion momentum distribution collimated to the target normal as an initial condition. As a comparison, the spectra for the ion acceleration collimated to the laser direction are also shown as dotted lines in Figs. 1 and 2. No possibility of the ion acceleration in the laser direction is clearly seen from the spectra fitting for both polarization conditions.

The well-fitted three-dimensional momentum distributions, corresponding to the dashed line in Figs. 1 and 2, are given by an anisotropic 3D Maxwellian distribution. The distributions projected on the $x-y$ plane are shown as a contour plot in Fig. 1(c) (s-polarization) and Fig. 2(c) ( $p$-polarization), where Px represents to the target normal direction, the Py axis parallel to the target surface and Pz the direction perpendicular to the figures. Each line represents every $10^{0.2}$ of the ion number in a logarithmic scale. The ion temperatures in the $x$ direction, corresponding to the target normal direction, are about $330 \mathrm{keV}$ for the $s$-polarization and $370 \mathrm{keV}$ for the $p$-polarization, whereas the temperature to the $y$ - and $z$ directions are about $30 \mathrm{keV}$ for the $s$-polarization and $40 \mathrm{keV}$ for the $p$-polarization. The results indicate the ions are accelerated toward the target normal for either linear polarization. We also changed the laser incident angles $\left(10^{\circ}-45^{\circ}\right)$ for the different polarization conditions. At all the incident angles and polarizations, we concluded that the ion acceleration was direct into the target along to target normal direction.

From the Monte Carlo fitting, the number of the accelerated ions to the target normal direction were order of $10^{12}$ per stradian (over $100 \mathrm{keV}$ ions) for both polarizations, corresponding to a few percent of the laser energy. The number was approximately equivalent to the number within the volumes of the spot size times the skin length.

Momentum distributions of accelerated ions inside the target are also investigated with a two-dimensional particlein-cell (PIC) code at different polarization ( $s$ - and $p$ polarization) in ultra-intense laser-plasma interactions. As the calculation conditions, the deuteron plasma is fully ionized and its geometry has an oblique surface to the laser axis set in the middle of a simulation box (23 $\mu \mathrm{m}$ square). The density profile has a steep exponential shape from $4 n_{c}$ as a maximum density down to $0.1 n_{c}$ with a scale length of 0.1 $\mu \mathrm{m}$. The $1.05 \mu \mathrm{m}$ laser light with a 200 fs pulse duration irradiates the plasma at an incidence angle of $30^{\circ}$ and a peak intensity of $10^{19} \mathrm{~W} / \mathrm{cm}^{2}$ during the simulation time within a $7 \mu \mathrm{m}$ spot diameter. Figure 3 shows the contours of the ion momentum for (a) $s$-polarization and (b) $p$-polarization at the end of pulse duration. Each contour line corresponds to every $10^{0.5}$ of the ion number in a logarithmic scale. At either polarization, the ion accelerations are collimated toward the target normal, which agrees with the experimental results.

\section{DISCUSSION}

All the experiment and simulation results show the ions are accelerated at the target surface in the directions of target normal into the target with both polarizations. These results indicate that the ions are not accelerated by a direct Coulomb potential between the accelerated electrons and the surface

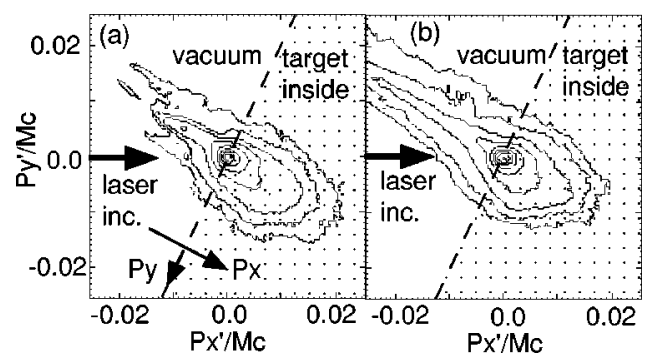

FIG. 3. The ion momentum distributions of (a) s-polarization and (b) $p$-polarization from 2D PIC simulation. The momentum coordinate is normalized by Mc. Each contour line corresponds to every $10^{0.5}$ of the ion number in a logarithmic scale.

ions. This is because the accelerated directions of the electrons are different and depend upon the polarization condition (target normal for $p$-polarization due to Brunel absorption; laser direction for $s$-polarization due to $\mathrm{J} \times \mathrm{B}$ acceleration), ${ }^{17}$ but possibly by the electrostatic field due to the charge displacement at the target front. A charge displacement will be generated along the target surface due to an exclusion of the fast electrons, which causes the creation of an electrostatic field to the surface normal direction. Then this static field accelerates ions to its direction, target normal, at any polarization condition.

On the other hand, the acceleration energy could be different between $s$-polarization and $p$-polarization. Table I shows the energy ratio among the $\mathrm{Px}, \mathrm{Py}$, and $\mathrm{Pz}$ direction of the ion momentum distribution and the energy to the Px direction from the experiment at the incident angle of $45^{\circ}$ and the PIC simulation $\left(30^{\circ}\right)$ results. The directions of the ion acceleration were always target normal direction. However, the energies of ions were clearly different from each other at different polarization. At the pure $p$-polarization condition with $45^{\circ}$ incidence, the ion temperature reduced to approximately $120 \mathrm{keV}$ whereas the $s$-polarized light created over $300 \mathrm{keV}$ temperature distribution. Similar behavior of the ion temperature was also observed from the PIC results.

The difference of the accelerated ion energy could be due to different plasma heating mechanisms at each laser polarization. Figure 4 shows the energy distribution of accelerated ions on the $x$-coordinate for (a) $s$-polarization and (b) $p$-polarization from the PIC simulation at the end of pulse duration. The laser and plasma parameters in the calculation are the same as those in Fig. 3. The positive direction on the

TABLE I. Summary of momentum distributions and temperature of ions from experiments (incident angle: $45^{\circ}$ ) and 2D PIC simulations $\left(30^{\circ}\right.$ ) at different polarization. Px is the momentum to the rear normal of the target surface, Py for $\mathrm{y}$ axis parallel to the target surface and $\mathrm{Pz}$ the direction orthogonal to the $x-y$ plane. Ex shows the temperature of accelerated ions to the $x$ direction (the target inside).

\begin{tabular}{cccc}
\hline \hline & Polarization & $\begin{array}{c}\text { Momentum ratio } \\
(\text { Px:Py:Pz })\end{array}$ & $\begin{array}{c}\text { Ex } \\
{[\mathrm{keV}]}\end{array}$ \\
\hline \multirow{2}{*}{ Exp. } & $s$-pol. & $2.2: 1: 1$ & 330 \\
& $p$-pol. & $1.4: 1: 1$ & 120 \\
Sim. & $s$-pol. & $2.8: 1: 1$ & 400 \\
& $p$-pol. & $1.4: 1: 1$ & 120 \\
\hline \hline
\end{tabular}




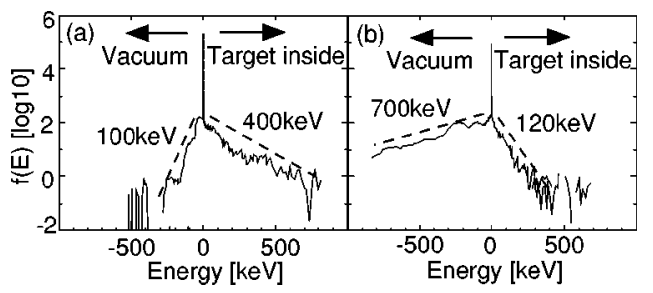

FIG. 4. The ion energy distributions accelerated to rear normal of the target surface for (a) $s$-polarization and (b) $p$-polarization from the PIC simulation. The positive direction of the transverse axis corresponds to the target inside and the negative to vacuum (target normal). The dotted lines show a nearMaxwellian distribution with a temperature of $400 \mathrm{keV}$ into the target and $100 \mathrm{keV}$ to vacuum at $s$-polarization. At $p$-polarization, the temperature into the target is 120 and $700 \mathrm{keV}$ to vacuum.

transverse axis corresponds to the target inside and the negative to the vacuum side. The temperature of the ions accelerated inside the target is about $400 \mathrm{keV}$ at the $s$-polarization and $120 \mathrm{keV}$ at the $p$-polarization. On the other hand, the temperature to the vacuum side at the $p$-polarization $(700$ $\mathrm{keV})$ is much larger than the temperature at $s$-polarization $(100 \mathrm{keV})$.

This result indicates a strong plasma expansion to the vacuum side due to the larger laser absorption at the $p$-polarization, such as resonance and Brunel absorption. ${ }^{18}$ From the PIC simulation, the absorption efficiency of $p$-polarized laser light at oblique irradiation is about three times larger than that of $s$-polarized light, i.e., $26 \%$ for $p$-polarization and $8 \%$ for $s$-polarization. ${ }^{19}$ The significant absorption at $p$-polarization creates higher "bulk" electron temperature and therefore the higher pressure, which prevents the ion acceleration to target inside. The electron motion can be described by the momentum equation ${ }^{20}$

$$
m_{e} n_{e}\left[\frac{\partial u_{e}}{\partial t}+\left(u_{e} \cdot \nabla\right) u_{e}\right]=q n_{e} \frac{\partial \varphi}{\partial z}+\frac{1}{c} j \times B-\nabla p_{e},
$$

where $m_{e}$ is the electron mass, $n_{e}$ the electron density, $u_{e}$ the electron velocity, $q$ the electric charge, and $p_{e}$ the electron pressure.

Assuming the ion mass $m_{i}$, ion number density $n_{i}$, and ion velocity $u_{i}$, ion motion can be represented by

$$
m_{i} n_{i}\left[\frac{\partial u_{i}}{\partial t}+\left(u_{i} \cdot \nabla\right) u_{i}\right]=q n_{i} \frac{\partial \varphi}{\partial z},
$$

along with the Poisson equation for the potential $\varphi$ generated by the charge displacement

$$
\frac{\partial^{2} \varphi}{\partial z^{2}}=-4 \pi e\left(n_{i}-n_{e}\right) .
$$

In the dense plasma $\left(n_{e} \gg n_{c}\right)$, the electron inertia can be neglected assuming the quasi neutrality between ions and electrons $\left(t \gg \omega_{p e}^{-1}, n_{i} \simeq n_{e}\right)$ and that the diffusion length due to electron-ion collision is much larger than the skin depth. ${ }^{21}$ Therefore, from Eqs. (1) and (2), the motion of ions can be given

$$
m_{i} n_{i} \frac{d v_{i}}{d t}=F_{\text {pond }} \cos \theta-F_{p} \sim(2-A) \frac{I}{c} \cos \theta \frac{1}{l_{s}}-\frac{n_{e} k T_{e}}{L},
$$

where $F_{\text {pond }}$ the ponderomotive force, $F_{p}$ the pressure force, $I$ the laser intensity, $A$ the absorption, $l_{s}$ the skin-depth, $\theta$ the incident angle, $T_{e}$ the electron temperature and $\mathrm{L}$ the density scale. At the intensity of $10^{19} \mathrm{~W} / \mathrm{cm}^{2}, F_{\text {pond }} \cos \theta / n_{i}$ is about $9.8 \times 10^{-3}$ [dyn] (s-polarization) and $8.9 \times 10^{-3}$ [dyn] $(p-$ polarization) at $\theta=45^{\circ}$ and $l_{s}=0.1 \mu \mathrm{m} .{ }^{21}$ On the other hand, $F_{p} / n_{i}$ at the relativistic critical density is $2.0 \times 10^{-3}$ [dyn] $\left(s\right.$-polarization) and $2.8 \times 10^{-3}$ [dyn] ( $p$-polarization) using the electron temperature of $1.2 \mathrm{MeV}$ ( $s$-polarization) and 2.3 $\mathrm{MeV}$ (p-polarization) from the PIC simulation, ${ }^{22}$ respectively. Therefore, the ion energy from Eq. (4) could be 3.3 $\mathrm{MeV}$ for the $s$-polarization and $1.0 \mathrm{MeV}$ for the $p$-polarization. This simple estimation indicates that the ion energy for the $s$-polarization become larger than that for the $p$-polarization, which is consistent with the experiment and simulation results.

\section{SUMMARY}

We have measured the laser polarization dependence of neutron energy spectra to study the ion acceleration mechanism in ultra-intense laser interactions with a solid target. From the neutron spectra at different viewing angles, ion acceleration indicates in the direction of the target normal at any polarization or incident angles. These results are consistent with the 2D PIC simulations, indicating the ion acceleration with a static electric field by a charge displacement along with the target surface.

It is interesting to consider the possibility of contribution of the accelerated ions on fast ignitor scenario. Assuming the density profile of the implosion plasma is a Gaussian distribution with the peak density of $300 \mathrm{~g} / \mathrm{cm}^{3}$ and the core diameter of $30 \mu \mathrm{m},{ }^{23}$ the ion energy should be ranged on tens $\mathrm{MeV}$ in order to reach and stop at the core. However, the ion temperature we observed was just $330 \mathrm{keV}$ Maxwellian, which means the total energy of accelerated ions integrated over $10 \mathrm{MeV}$ was only order of $10^{-10} \mathrm{~J}$ at our $50 \mathrm{~J}$ laser experiment. If the ion temperature increases over a few $\mathrm{MeV}$, the energy fraction over $10 \mathrm{MeV}$ will raise up to $10 \%$ of the total ion energy. Therefore, assuming that the ion temperature scales with laser intensity in the same way as the hot electrons, ${ }^{24}$ laser intensity over $5 \times 10^{20} \mathrm{~W} / \mathrm{cm}^{2}$ will satisfy the condition. From the conversion efficiency of laser energy to the ions, the contribution to heating may become a few $\%$ of the fast electrons and will rapidly increase over the intensity.

\section{ACKNOWLEDGMENTS}

We acknowledge all the technical support at Institute of Laser Engineering for the laser operation, the target fabrication and the data acquisition. We especially thank Dr. T. Norimatu, Dr. H. Fujita, Dr. E. Yoshida, K. Sawai, T. Kawasaki, T. Matsuo, K. Suzuki, Y. Kimura, O. Maekawa, and T. Komenou.

${ }^{1}$ G. Malka and J. L. Miquel, Phys. Rev. Lett. 77, 75 (1996).

${ }^{2}$ K. B. Wharton, S. P. Hatchett, S. C. Wilks et al., Phys. Rev. Lett. 81, 822 (1998).

${ }^{3}$ A. P. Fews, P. A. Norreys, F. N. Beg et al., Phys. Rev. Lett. 73, 1801 (1994). 
${ }^{4}$ M. Tabak, J. Hammer, M. E. Glinsky et al., Phys. Plasmas 1, 1626 (1994). ${ }^{5}$ M. H. Key, M. D. Cable, T. E. Cowan et al., Phys. Plasmas 5, 1966 (1998).

${ }^{6}$ S. P. Hatchett, C. G. Brown, T. E. Cowan et al., Phys. Plasmas 7, 2076 (2000).

${ }^{7}$ A. J. Mackinnon, Y. Sentoku, P. K. Patel et al., Phys. Rev. Lett. 88, 215006 (2002)

${ }^{8}$ T. Ditmire, J. Zweiback, V. P. Yanovsky, T. E. Cowan, G. Hays, and K. B. Wharton, Nature (London) 398, 489 (1999).

${ }^{9}$ A. Zhidkov, M. Uesaka, A. Sakai, and H. Daido, Phys. Rev. Lett. 89, 205002 (2002).

${ }^{10}$ P. A. Norreys et al., Plasma Phys. Controlled Fusion 40, 175 (1998).

${ }^{11}$ L. Disdier, J.-P. Garçonnet, G. Malka, and J.-L. Miquel, Phys. Rev. Lett. 82, 1454 (1999).

${ }^{12}$ Y. Kitagawa et al., Fusion Eng. Des. 44, 261 (1999).

${ }^{13}$ Y. Kato et al., Plasma Phys. Controlled Fusion 39, A145 (1997).

${ }^{14}$ N. Izumi, K. Yamaguchi, T. Yamagajo et al., Rev. Sci. Instrum. 70, 1221 (1999).
${ }^{15}$ J. D. Jackson, Classical Electrodynamics, 2nd ed. (Wiley, New York, 1975), Chap. 13.

${ }^{16} \mathrm{R}$. J. Howerton et al., Index to the LLNL Evaluated Charged-Particle Library (Lawrence Livermore National Laboratory, LLNL, 1986).

${ }^{17}$ H. Habara, Ph.D. thesis, University of Osaka, 2000; Y. Toyama et al., Phys. Rev. Lett. (submitted).

${ }^{18}$ F. Brunel, Phys. Rev. Lett. 59, 52 (1987).

${ }^{19}$ Y. Sentoku, H. Ruhl, K. Mima et al., Phys. Plasmas 6, 2855 (1999).

${ }^{20}$ W. L. Kruer, The Physics of Laser Plasma Interactions (Addison-Wesley, New York, 1987), p. 118.

${ }^{21}$ E. G. Gamaly, Phys. Fluids B 5, 3765 (1993).

${ }^{22}$ Y. Sentoku, K. Mima, S. Kojima, and H. Ruhl, Phys. Plasmas 7, 689 (2000)

${ }^{23}$ S. Atzeni, Phys. Plasmas 6, 3316 (1999).

${ }^{24}$ S. C. Wilks, W. L. Kruer, M. Tabak, and A. B. Langdon, Phys. Rev. Lett. 69, 1383 (1992) 